\title{
MANa1-2MAN decorated liposomes enhance the immunogenicity induced by a DNA vaccine against BoHV-1
}

Kornuta, Claudia Alejandra 1,2; Bidart, Juan Esteban 1,2; Soria, Ivana 1; Gammella, Mariela 1; Quattrocchi, Valeria 1; Pappalardo, Juan Sebastián 4; Salmaso, Stefano 5; Torchilin, Vladimir Petrovich ${ }^{6}$; Cheuquepán Valenzuela, Felipe ${ }^{2,7}$; Hecker, Yanina ${ }^{\mathbf{2 , 7}}$; Moore, Dadin Prando ${ }^{\mathbf{2}, 7}$; Zamorano, Patricia Inés ${ }^{\mathbf{1 2 , 3}}$; Langellotti, Cecilia Ana* 1,2

${ }^{1}$ Instituto de Virología e Innovaciones Tecnológicas (IVIT, INTA-CONICET), Hurlingham, Buenos Aires, Argentina

${ }^{2}$ Consejo Nacional de Investigaciones Científicas y Técnicas, Buenos Aires, Argentina

${ }^{3}$ Universidad del Salvador, Buenos Aires, Argentina

4 Instituto de Investigaciones Forestales y Agropecuarias Bariloche (IFAB, INTA-CONICET), San Carlos de Bariloche, Río Negro, Argentina

${ }^{5}$ Department of Pharmaceutical and Pharmacological Sciences, Universita degli Studi di Padova, Padova PD, Italy

${ }^{6}$ Center for Pharmaceutical Biotechnology and Nanomedicine, Northeastern University, Boston, MA, USA

${ }^{7}$ EEA Balcarce, Instituto Nacional de Tecnología Agropecuaria (INTA), Balcarce, Buenos Aires, Argentina

\section{*Corresponding author:}

Dra. Cecilia Langellotti

E-mail: langellotti.cecilia@inta.gob.ar

\section{Abstract}

New technologies in the field of vaccinology arise as a necessity for the treatment and control of many diseases. Whole virus inactivated vaccines as well as modified live virus ones used against Bovine Herpesvirus-1 (BoHV-1) infection have several disadvantages. Previous works on DNA vaccines against BoHV-1 have demonstrated the

This article has been accepted for publication and undergone full peer review but has not been through the copyediting, typesetting, pagination and proofreading process, which may lead to differences between this version and the Version of Record. Please cite this article as doi: 10.1111/tbed.13718

This article is protected by copyright. All rights reserved 
capability to induce humoral and cellular immune responses. Nevertheless, "naked" DNA induces low immunogenic response. Thus, loading of antigen encoding DNA sequences in liposomal formulations targeting dendritic cell receptors could be a promising strategy to better activate these antigen presenting cells (APC). In this work, a DNA based vaccine encoding the truncated version of BoHV-1 glycoprotein D (pCIgD) was evaluated alone and encapsulated in a liposomal formulation containing LPS and decorated with MAN 1 1-2MAN-PEG-DOPE (pCIgDMan-L). The vaccinations were performed in mice and bovines. The results showed that the use of pCIgD-Man-L enhanced the immune response in both animal models. For humoral immunity, significant differences were achieved when total antibody titers and isotypes were assayed in sera.

Regarding cellular immunity, a significant increase in the proliferative response against BoHV1 was detected in animals vaccinated with pCIgD-Man-L when compared to the response induced in animals vaccinated with pCIgD. In addition, upregulation of CD40 molecules on the surface of bovine dendritic cells (DCs) was observed when cells were stimulated and activated with the vaccine formulations. When viral challenge was performed, bovines vaccinated with MAN 1 1-2MAN-PEG-DOPE elicited better protection which was evidenced by a lower viral excretion.

These results demonstrate that the dendritic cell targeting using MAN 1 1-2MAN decorated liposomes can boost the immunogenicity resulting in a long-lasting immunity.

Liposomes decorated with MANa1-2MAN-PEG-DOPE were tested for the first time as a DNA vaccine nanovehicle in cattle as a preventive treatment against BoHV-1. These results open new perspectives for the design of vaccines for the control of bovine rhinotracheitis.

Keywords: adjuvant, BoHV-1, DNA, DC targeting vaccine, liposome

\section{Introduction}

Bovine herpesvirus 1 (BoHV-1), an enveloped virus belonging to the alphaherpesvirus subfamily, infects cattle of all ages and breeds worldwide (Babiuk et al., 1987; Tikoo et al., 1995). This virus is a pathogen whose infection can severely impact in cattle production. It causes a variety of symptoms in cattle including infectious bovine rhinotracheitis (IBR), conjunctivitis, abortions and shipping fever, which is a complicated infection of the upper respiratory tract (Jones, 2003). The pathogenesis is responsible for considerable economic losses due to decreased milk production, weight loss and abortion and has been recognized as an important component of the bovine respiratory complex.

BoHV-1 initiates the disorder through immunosuppression that could render the animals more susceptible to secondary bacterial infections, leading to pneumonia and occasionally to death. BoHV-1 establishes latency. Latently infected animals should always be considered a potential source of infection (Bitsch, 1973), although vaccination can considerably reduce the amount of virus excreted following reactivation (Mars et al., 2001).

In general, there are control programs including the use of conventional inactivated vaccines or attenuated living vaccines but there are no specific international programs to eradicate BoHV-1. In Europe, only a small number of countries achieved the goal of IBR-eradication (Blickenstorfer et al., 2010), by means of slaughtering a great number 
of healthy, seropositive animals because they are persistently infected with BoHV-1. In other European countries, a strategy to control this virus has been the implementation of vaccinations with live and inactivated gE-deleted BoHV1 (van Drunen Littel-van den Hurk, 2006; Romera et al., 2014). In countries where this infection is endemic such as Argentina, Brazil and Spain, voluntarily intensive vaccination programs are implemented to reduce the prevalence of infected animals.

Vaccination remains one of the most cost-effective strategies to prevent and control the clinical signs and transmission of these viruses. Nevertheless, these vaccines have a series of disadvantages for BoHV-1. Although live attenuated vaccines confer protection, there is a risk of reversion to virulence, induction of abortions in pregnant cows and reexcretion of the vaccine strain by immunosuppression of cattle. In the case of inactivated vaccines, there is a lack in providing a complete protection and these vaccines are generally poor inducers of cellular immunity (Deshpande et al., 2002; Blome et al., 2013; Quattrocchi et al., 2017).

BoHV-1 uses a variety of mechanisms to elude the host's immune system. By spreading intracellularly, it can survive in the presence of antiviral specific antibodies (Fuller and Lee, 1992; Miethke et al., 1995). For this reason, cytotoxic T-lymphocytes (CTL) are critical for the complete viral clearance (Langellotti et al., 2011).

DNA vaccines emerge as an alternative to conventional vaccines. These vaccines have the potential to induce both cellular and humoral immune responses against an antigen encoded by recombinant DNA (Kanthesh et al., 2018) and are highly specific; the expressed immunizing antigen is subject to the same modifications as in a natural infection (Lee et al., 2015) without the risk of pathogenicity due to incompletely inactivated virus or due to reverted attenuated virus (Rajčáni et al., 2005). The use of naked DNA vaccines in small animal models possess and adjuvant or immunomodulator effect itself (Yamamoto et al., 1992; Krieg et al., 1995) but in large animals the conferred protection is inefficient. For this reason, in order to improve and enhance the immune responses there are attempts using DNA plasmids formulated with adjuvants and delivery systems (Quattrocchi et al., 2017).

The aim of this study was to evaluate the immune responses elicited in a mice model and in bovines by a plasmid encoding the truncated version of BoHV-1 glycoprotein D (pCIgD) vehicularized in liposomes decorated with a ligand that specifically targets dendritic cells (Man-L). The bilayer formulation included LPS as an adjuvant to stimulate the immune response. The liposome surface decoration was performed using a patented synthetic molecule (Pappalardo et al., 2013), whose specific $\alpha 1$,2Mannobiose ligand has an affinity for DC-SIGN (Feinberg et al., 2007; Yamakawa et al., 2008). The MAN 1 1-2MAN-PEG-DOPE derivative was selected because it can target dendritic cells (DCs) of various species (Pappalardo et al., 2013) and thus provide selective delivery of different cargoes such as antigens and nucleic acids that can be uptaken and processed for presentation in the contexts of MHC-I and II. Brucella ovis HS antigen, which contains a short polysaccharide LPS and some OMP proteins, is associated to this nanovaccine as an adjuvant (LPS). The formulation of DNA loaded nanovehicles confers advantages such as an increased cargoe uptake by antigen presenting cells (APCs), cytokine secretion stimulated by APC or lymphocytes, increased cargoe stability and decreased cargoe degradation (Zaman et al., 2013). MAN 1 1-2MAN-PEG-DOPE has been proven to specific target mouse and human primary DC cultures in vitro (Pappalardo et al., 2013). Previous studies have demonstrated in vivo the improved delivery of LPS as an antigen to DC by anchoring MANa1-2MANPEG-DOPE on the liposome surface in a nanovaccine formulation and the effectiveness of this DC-targeted nanovaccine against Brucella ovis in rams (Pappalardo et al., 2016). 
DC-targeted liposomes using MAN 1 1-2MAN-PEG-DOPE were for the first time formulated as a DNA nanovaccine against BoHV-1 and evaluated in mice and bovines. These results could be useful to design and improve a vaccine for the control of IBR.

\section{Materials and Methods}

\subsection{DNA Plasmid generation}

The pCI-neo mammalian expression vector (pCIneo) was obtained from Promega (Madison, WI, USA) and the gene encoding the truncated version of BoHV-1 glycoprotein D was cloned (pCIgD) by a standard procedure previously described (Langellotti et al., 2011). The plasmids were amplified in transformed MAX Efficiency ${ }^{\mathrm{TM}}$ DH5 $\alpha$ Competent E. coli from Invitrogen (Waltham, MA, USA), and purified by anion exchange chromatography using the Plasmid Giga Kit purchased from Qiagen (Hilden, NRW, Germany). The integrity and concentration of the plasmids were evaluated before and after each vaccine formulation where restriction digests and 260/280 absorbance ratios were analyzed.

\subsection{Liposome formulation}

A lipid film was prepared by rotary evaporation from a mixture of phosphatidylcholine (PC), cholesterol (Chol), 1,2dioleoyl-3-trimethylammonium-propane (DOTAP), Brucella ovis HS extract (LPS), and the $\alpha 1,2-M a n n o b i o s e-$ $\mathrm{PEG}_{2 \mathrm{kDa}}$-DOPE derivative (Man $\alpha$ ) (Pappalardo et al., 2013) in chloroform. PC, Chol and DOTAP were purchased at Avanti Polar Lipids (Alabaster, AL, USA). The B. ovis HS extract was kindly provided by Mgtr. Carlos Robles (Robles, 2009). The lipid film was rehydrated for 30 minutes in sterile normal saline $(0.9 \% \mathrm{NaCl}), \mathrm{pH} 7.4$, at a PC concentration of $1.2 \mathrm{mg} / \mathrm{mL}$ (mice vaccines) or $4.8 \mathrm{mg} / \mathrm{mL}$ (bovine vaccines) in the final suspension and then vortexed for 5 minutes. The liposomes were prepared by extrusion of the suspension through a $200 \mathrm{~nm}$ pore Whatman ${ }^{\circledR}$ Nucleopore ${ }^{\circledR}$ Polycarbonate membrane using an Avanti Polar Lipids Mini Extruder ${ }^{\mathrm{TM}}$.

During liposome formulation and extrusion, the combination of all components forming the liposomal suspension, is passed through the 200nm pore polycarbonate filter which will generate a uniform population of liposomes. The liposomes containing MANa1-2MAN-PEG-DOPE were extensively characterized (Pappalardo et al., 2013) and the average size is around $100 \mathrm{~nm}$, when extruded through a $200 \mathrm{~nm}$ filter. All lipids remain in the lipid bilayer, including the LPS. The spikes of PEG with the dimannose ends will protrude outside the liposome core. The DNA is entrapped around the outer bilayer by the effect of the cationic DOTAP -which strongly binds the anionic plasmid DNA-. The plasmids then are attached to the outer surface and covered/protected by the PEG spikes that protrude further (that is why liposomes with PEG are also known as stealth liposomes).

For mice vaccines: formulation of Man-L had PC:Chol:DOTAP:LPS:Man $\alpha$ with a 60:30:10:4.3:2 molar ratio [2.13 $\mathrm{mg} / \mathrm{mL}$ total lipids]. These were incubated $\mathrm{ON}$ with plasmids resulting in a final dose of $15 \mu \mathrm{g}$ DNA/300 $\mu \mathrm{L}$ liposomes. 
For bovine vaccines: formulation of Man-L had PC:Chol:DOTAP:LPS:Man $\alpha$ with a 60:30:30:4.3:2 molar ratio [9.96 $\mathrm{mg} / \mathrm{mL}$ total lipids]. These were incubated ON with plasmids, resulting in a final dose of $600 \mu \mathrm{g}$ DNA/1500 $\mu \mathrm{L}$ liposomes. "Bovine vaccine" was formulated with a higher ratio of DOTAP with respect to "mice vaccine" to increase liposome stability due to the higher concentration of lipids in medium.

\subsection{Vaccine formulations and animal treatment}

Adjuvanted PC:Chol:DOTAP:LPS:Man $\alpha$ liposome (Man-L) was included as follows.

In mice: vaccines were formulated using a $15 \mu \mathrm{g}$ dose of pCIgD according to previous dose-response results (Langellotti et al., 2011) or pCIneo as negative control. Groups of BALB/c $(n=5)$ were intradermally (i.d.) inoculated at 0 and 20 days with $15 \mu \mathrm{g} / 0.3 \mathrm{~mL}$ of pCIgD or pCIneo alone; $15 \mu \mathrm{g} / 0.3 \mathrm{ml}$ of pCIgD-Man-L or pCIneo-Man-L.

In bovines: a dose of $600 \mu \mathrm{g}$ pCIgD was chosen based on a dose-response curve (data not shown). Seronegative bovines to BoHV-1 (1-2 years old) were vaccinated i.d. in the back of the ear with $600 \mu \mathrm{g} / 1,5 \mathrm{~mL}$ (distributed in five sites) of pCIgD $(n=5)$, pCIgD-Man-L $(n=5)$ and pCIneo as negative control $(n=4)$. Bovines were vaccinated at 0 , 30 and 60 days.

\subsection{Enzyme-linked immunosorbent assay for detection of anti-BoHV-1 antibodies}

Before each animal vaccination, sera were tested by anti BoHV-1 enzyme-linked immunosorbent assay (ELISA). Briefly, Immulon 1B polystyrene microtiter plates from Thermo Fisher Scientific Inc. (Waltham, MA, USA) were coated with inactivated BoHV-1 (iBoHV-1) Los Angeles strain (1/300) in 0.1 M carbonate-bicarbonate buffer, pH 9.6 and incubated overnight $(\mathrm{ON})$ at $4^{\circ} \mathrm{C}$. Plates were blocked with PBS $0.05 \%$ Tween 20 (PBST) containing $1 \%$ ovalbumin (PBST-OVA). Serial dilutions of mice or bovines sera were prepared in PBST-OVA and dispensed in 50 $\mu \mathrm{L} /$ well. Plates were washed three times with PBST-OVA and incubated with anti-mouse or anti-bovine IgG peroxidase conjugate from KPL (Gaithersburg, MD, USA) respectively for $1 \mathrm{~h}$ at $37^{\circ} \mathrm{C}$. After extensive washing with PBST, ortho-phenylene-diamine (1,2-benzenediamine) dihydrochloride -OPD- (Sigma-Aldrich, St. Louis, MO, USA) and $\mathrm{H}_{2} \mathrm{SO}_{4}$ were added as substrate. To measure the absorbance at $492 \mathrm{~nm}$ a Multiskan MS/352 microplate reader was used (Labsystems Oy, Helsinki, Finland). The cut-off was established using the negative sera mean at A492 plus 2 standard deviations (SD). Titers were calculated as the $\log _{10}$ of the reciprocal value of the highest dilution that had an OD above the cut-off level from animals vaccinated with pCIneo.

\subsection{Immunoglobulin isotyping ELISA}

Isotype profile by indirect ELISA was evaluated to determine the IgG1, IgG2a, IgG2b and IgG3 subtypes in immunized mice at 40 days post vaccination (dpv). The IgG1 and IgG2 isotype titer from sera of immunized bovines was determined at $90 \mathrm{dpv}$, and the IgA from nasal swabs was determined at 6 days post challenge (dpc). Briefly, Microlon polystyrene microtiter plates from Greiner Bio-One GmbH (Kremsmünster, OÖ, Austria) were coated with iBoHV-1 and dilutions were loaded as described previously. Specific antibodies in mice sera were detected with 
biotinylated goat anti-mouse IgG1, IgG2a, IgG2b and IgG3 from Caltag Laboratories (San Francisco, CA, USA). After a 60 minutes incubation, plates were washed with PBST and a dilution of streptavidin/alkaline phosphatase (Sigma-Aldrich) was added to each well. The 1/50 sera dilution OD readings at A492 nm corresponding to IgG1, $\operatorname{IgG2a}, \operatorname{IgG} 2 \mathrm{~b}$, and $\operatorname{IgG} 3$ were recorded.

For isotype detection in bovines, the ELISA described in chapter 2.4 with modifications was used. An anti-bovine IgG1, IgG2, or IgA mouse monoclonal antibodies were used (kindly provided by Dr. S. Srikumaran from the University of Nebraska, USA). An incubation with horse radish peroxidase (HRP) conjugated anti-mouse Ig was followed. Finally, the reaction was visualized, and the cut-off for IgG1 and IgG2was established as described before. The results for IgA 1/2 dilution in nasal swabs were expressed as OD at A492 nm.

\subsection{Afferent lymph dendritic cell (ALDC) stimulation in vitro}

The extraction of ALDCs from bovines was performed as previously described (Hope et al., 2006) by cannulation of pseudo afferent lymph vessels. Dendritic cells were incubated in RPMI 1640 medium supplemented with $10 \%$ fetal bovine serum from Gibco (Thermo Fisher Scientific Inc.), 1\% penicillin-streptomycin-amphotericin B antibioticantimycotic solution (Sigma-Aldrich) and $50 \mathrm{mM}$ 2-mercaptoethanol (RPMI 10\% FBS). Treatments were mock stimulated or $1 \mu \mathrm{g} / \mathrm{mL}$ pCIgD, pCIgD-Man-L vaccine formulations or plasmid free $\alpha 1,2-M_{a n n o b i o s e-P E G}$ 2kDa $-\mathrm{DOPE}$ decorated liposome (Man-L) with (+) or without (-) LPS from Brucella ovis. A direct cell surface staining was performed after a $16 \mathrm{~h}$ incubation with a monoclonal antibody DEC205-FITC from AbD Serotec (Kidlington, Oxfordshire, UK). Indirect surface staining was performed using monoclonal antibody anti-CD40 (AbD Serotec), and anti-mouse IgG PE conjugated from Jackson ImmunoResearch Laboratories, Inc. (West Grove, PA, USA). Finally, fixed cells $(0.2 \%$ paraformaldehyde) were acquired using a Becton Dickinson FACScalibur flow cytometer and CellQuest software (San Jose, CA, USA).

\subsection{Mouse splenocytes and bovine peripheral blood mononuclear cell (PBMC) isolation}

Splenocytes from immunized mice were collected at $120 \mathrm{dpv}$, stimulated in vitro with iBoHV-1, and assayed for antigen-specific proliferation. Splenocytes were obtained by gentle homogenization of spleens in RPMI 10\% FBS, and counted using Turk method. Venipuncture was performed to collect bovine blood samples using syringes containing sodium citrate as an anticoagulant. Ficoll-Paque ${ }^{\text {TM }}$ PLUS (density $1.077 \mathrm{~g} / \mathrm{mL}$ ) from GE Healthcare (Chicago, IL, USA) was used as described previously (Romera et al., 2000) to isolate PBMCs by centrifugation. Cells were counted using Trypan blue.

\subsection{Lymphoproliferation Assay}

Carboxyfluorescein diacetate succinimidyl ester (CFSE) $3 \mu \mathrm{M}$ was incubated with the cell cultures for $30 \mathrm{~min}$ at $37^{\circ} \mathrm{C}$ to label splenocytes and PBMCs suspensions. Later, cells were washed and resuspended in RPMI $10 \%$ FBS as described previously (Quattrocchi et al., 2017). A concentration of 5x105 CFSE-labeled PBMCs or splenocytes per 
well were added to a 96-well U-bottom plate. In each well, $100 \mu \mathrm{L}$ of complete medium containing iBoHV-1 $(\mathrm{MOI}=1)$, concanavalin A (ConA) (Sigma-Aldrich) as positive proliferation control, or medium as negative control were added. After cell incubation for 4 days (mice) or 5 days (bovines) at $37^{\circ} \mathrm{C}$ in $5 \% \mathrm{CO}_{2}$ atmosphere, cells were harvested and fixed and the proliferation was analyzed by flow cytometry as described in chapter 2.6.

\subsection{Viral challenge in bovines}

At 90 dpv, bovines were challenged by aerosol exposition as described previously (Romera et al., 2014; Quattrocchi et al., 2017) with $5 \mathrm{~mL}$ of $1 \times 10^{6.81} \mathrm{TCID}_{50} / \mathrm{mL}$ BoHV-1 Los Angeles strain in each nostril. Animals were clinically examined at $0,3,5,6,7,8$ and $12 \mathrm{dpc}$. Rhinitis severity was scored after viral challenge as follows: $0=$ absence of rhinitis ; $1=$ slight serous rhinitis with or without mild serous conjunctivitis; $2=$ severely serous rhinitis; $3=$ seromucous with moderate or severe conjunctivitis; 4 = mucopurulent rhinitis with or without conjunctivitis (Romera et al., 2014).

Samples for viral excretion were obtained at the same days described above by inserting tampons into each nostril as nasal swabs. These tampons were dipped in Minimal Essential Medium (MEM) which contained 5,000 IU/mL penicillin, 2,500 $\mu \mathrm{g} / \mathrm{mL}$ streptomycin, and $10 \mu \mathrm{g} / \mathrm{mL}$ amphotericin B. These samples were inoculated onto MDBK cell monolayers in serial dilutions, and checked for cytopathic effect (cpe). An end point dilution method was performed to obtain virus titers (Reed and Muench, 1938).

\subsection{Statistical analysis}

ANOVA and Bonferroni post-hoc tests were performed to evaluate if the differences were significant, using the InfoStat software. Differences between two means were analyzed using Student's t-test. A value of $\mathrm{P}<0.05$ was considered as statistical significant.

\section{Results}

\section{1 pCIgD-Man-L increased specific BoHV-1 antibodies in mouse}

In order to study the humoral immune response induced by pCIgD-Man-L vaccine, an ELISA test to detect antiBoHV-1 antibodies was used. Vaccines pCIgD and pCIgD-Man-L were capable to induce specific antibodies at 20 and 40 days after administration, but the formulation with liposomes improved notably the IgG titer with respect to $\mathrm{pCIgD}(\mathrm{p}<0.01, \mathrm{p}<0.05$ respectively). In addition, it was possible to detect titers at 120 days. For pCIgD-Man-L, antibody titer levels remained higher $(\mathrm{p}<0.001)$ with respect to those observed on the $\mathrm{pCIgD}$ treated group, where the titer declined (Fig.1a).

When the profile of antibody isotypes was analyzed, a marked difference between the group treated with pCIgD-Man$\mathrm{L}$ and the group treated with $\mathrm{pCIgD}$ alone was observed. The group treated with pCIgD-Man-L had elevated IgG1 $(\mathrm{p}<0.001), \operatorname{IgG} 2 \mathrm{a}(\mathrm{p}<0.05)$ and $\operatorname{IgG} 2 \mathrm{~b}(\mathrm{p}<0.01)$ levels with respect to the group treated with $\mathrm{pCIgD}(\mathbf{F i g} .1 \mathrm{~b})$. 


\section{2 pCIgD-Man-L induces viral specific proliferation of lymphocytes in mice}

A lymphoproliferative assay was performed to study the cellular immune response against the viral antigen. After splenocyte collection from mice, the cells were stimulated in vitro with iBoHV-1. As a result, the percentages of specific lymphoproliferation was higher in pCIgD-Man-L group $(\mathrm{p}<0.05)$ in comparison with the pCIgD group (Fig.2). Taken together, these results indicate that $\mathrm{pCIgD}$ vaccine formulated with liposome Man-L could induce a higher humoral and cellular response against BoHV-1 in mice. Therefore, this formulation was further tested in a trial in bovines, the natural host species.

\section{3 pCIgD-Man-L increased specific BoHV-1 antibodies titers in bovines}

The animals were vaccinated at 0,30 and 60 days. Regarding antibody kinetics, pCIgD and pCIgD-Man-L were able to induce specific anti-BoHV-1 antibody levels (Fig.3a). However, significant differences in pCIgD-Man-L at $60 \mathrm{dpv}$ $(\mathrm{p}<0.05)$ were found compared with $\mathrm{pCIgD}$ group. After viral challenge $(90 \mathrm{dpv})$ all animals seroconverted (data not shown).

In addition, ELISA isotype titers at $90 \mathrm{dpv}$ against BoHV-1 were analyzed (Fig. 3b). Interestingly, there were significant differences in IgG1 $(\mathrm{p}<0.001)$ for the $\mathrm{pCIgD}$ and $\mathrm{pCIgD-Man-L}$ group versus $\mathrm{pCIneo}$, but in $\operatorname{IgG2}$ difference was observed only for $\mathrm{pCIgD-Man-L}(\mathrm{p}<0.05)$ with respect to the $\mathrm{pCIneo} \mathrm{group.}$

No significant differences among groups were found when neutralizing antibodies were measured; however, a trend of increasing neutralizing antibodies was observed in $\mathrm{pCIgD-Man-L}$ over pCIgD treated groups (data not shown).

Antibodies in nasal swabs were also evaluated. It is reported that the presence of antibodies in nasal mucosa confer protection against respiratory viral penetration. IgA isotype anti-BoHV-1 level in nasal swabs at 6 days post challenge (dpc) was evaluated (Fig. 3c). The results showed that the IgA titer in swabs of the pCIgD-Man-L treated group was significantly different compared with $\mathrm{pCIneo}$ treated group $(\mathrm{p}<0.05)$.

\section{4 pCIgD and Man-L modulate Dendritic Cells by Activation in vitro}

The key initiators of antiviral responses are dendritic cells, the major antigen presenting cell (APC) in the immune system (Ludewig et al., 2000). They endocytose, process and present antigens to naive $\mathrm{T}$ cells and act as messengers between the adaptive and innate immune responses. Afferent lymph dendritic cells (ALDCs) from bovines were used to elucidate if DNA alone or with loaded targeted liposomes were able to activate dendritic cells. ALDCs were incubated with vaccines pCIgD, pCIgD-Man-L (with or without LPS from Brucella ovis) or Man-L alone (with or without LPS from Brucella ovis) in vitro. CD40 surface molecules were significantly upregulated with respect to mock for $\mathrm{pCIgD}(\mathrm{p}<0.01)$, pCIgD-Man-L with $(\mathrm{p}<0.05)$ and without LPS $(\mathrm{p}<0.01)$. Also, CD40 was upregulated when treated with Man-L with LPS $(p<0.001)$ but not with Man-L without LPS. These results indicate that $\mathrm{pCIgD}$ alone and liposomes including LPS can activate dendritic cells (Fig.4).

3.5 pCIgD-Man-L induces BoHV-1 specific proliferation of lymphocytes 
Lymphoproliferation was evaluated at $12 \mathrm{dpc}$ in PBMCs after in vitro stimulation with inactivated BoHV-1. The results showed that pCIgD-Man-L treated group underwent the highest degree of proliferation with respect pCIneo $(\mathrm{p}<0.01)$ and $\mathrm{pCIgD}$ group $(\mathrm{p}<0.05)$ (Fig. 5). This result indicates that the liposomes possess a remarkable "adjuvant" effect and are capable of enhancing the cellular immune response elicited by $\mathrm{pCIgD}$ in bovines.

\subsection{Viral excretion is decreased in pCIgD-Man-L group}

Bovines were challenged at $90 \mathrm{dpv}$ via aerosol with BoHV-1 Los Angeles strain. In order to measure the viral excretion after virus challenge trial, nasal secretions were obtained.

Here it is shown that $\mathrm{pCIgD}$ and pCIgD-Man-L treatments were capable to reduce the viral excretion compared with the $\mathrm{pCIneo}$ treated group but the bovines that received the pCIgD-Man-L nanovaccine presented a markedly reduction of the viral excretion at $5 \mathrm{dpc}$ compared with pCIneo group $(\mathrm{p}<0.01)$. This phenomenon was not observed in the pCIgD group (Fig 6). In addition, these results were analyzed as the area under curve (AUC) of the viral titer. In this way, AUC of the viral titers in animals treated with pCIgD-Man-L was the lowest with respect to the other groups.

\section{7 pCIgD and pCIgD-Man-L presented similar clinical score}

Clinical score was obtained by measuring the symptoms acquired by the animals after the viral challenge and was established according with a degree of rhinitis ranging from 0 to 4 in ascending order of severity as we described in chapter 2.9. Therefore, the animals vaccinated with $\mathrm{pCIgD}$ and $\mathrm{pCIgD-Man-L}$ had a lower clinical score and there were significant differences compared with pCIneo group ( $\mathrm{p}<0.05$ ) at $6 \mathrm{dpc}$ for $\mathrm{pCIgD}$ and at $7 \mathrm{dpc}$ for pCIgD-Man-L $(p<0.05)$ but there were no significant differences between them (Fig. 7). Clinical score AUC was analyzed and again pCIgD-Man-L treated animals had the lowest level with respect to the other groups.

\section{Discussion}

DNA vaccines have taken great interest in the field of vaccinology because of their simplicity, safety, stability, and versatility for development (Kanthesh et al., 2018). Previous studies with these vaccines have shown to induce good levels of humoral and cellular immunity in different species models depending on the type of encoded antigen, route of administration and promoter efficiency (Huang et al., 2005). The glycoprotein D is involved in virus penetration and has been considered the major target in vaccine development against bovine and human herpesvirus. A big issue of DNA vaccines is the low immunogenicity due to difficulties in delivering DNA plasmids into the host cells for further synthesis of the encoded antigenic protein (Suschak et al., 2017). Here, the use of suitable delivery systems and specific molecular activators can be combined to generate adjuvants that can help to improve the efficiency of DNA vaccines. In the search to set up a vaccine that generates both types of immunity against BoHV-1, we investigated DNA vaccines using a DNA sequence encoding for the truncated version of BoHV-1 D glycoprotein (pCIgD). Our previous studies using the same DNA plasmid combined with chemical adjuvants in mice provided good protection evidences (Zamorano et al., 2002; Langellotti et al., 2011; Di Giacomo et al., 2015) while studies in 
cattle with a subsequent viral challenge showed the protection was only partial (Quattrocchi et al., 2017) which prompted us to further search for new formulation strategies.

In this context, we have designed a DNA nanovaccine using liposomes decorated with mannose molecules for DC targeting which is expected to enhance the transfection efficiency in DCs, including LPS in the formulation as a molecular adjuvant to promote DC maturation and immunity through TLR activation. This will result in the ability of the liposomes to selectively target the DC-SIGN receptor in DCs and deliver its cargoe. Liposomes were selected because they represent a self-assembling carrier that allow to combine different functional components on their surface resulting in a cooperative adjuvant effect. In addition, liposomes protect DNA from deoxyribonuclease attack (Gregoriadis et al., 2002).

The intradermal inoculation is one of the best performing administration strategies to deliver a DNA vaccines (Zonouzi et al., 2016). The rational for this is that the skin possess associated lymphoid tissue, including Langerhans cells, dendritic cells, keratinocytes and other immune cells. Here, we inoculated animals by the intradermal route, and we observed an improved humoral and cellular immune response. In agreement with evidences that truncated glycoprotein D can induce good levels of humoral immune response (Van Drunen Littel-van den Hurk et al., 1998; Lewis et al., 1999; Huang et al., 2005) we obtained antibody titers upon administration of pCIgD which was significantly increased when the plasmid encoding for the truncated glycoprotein D was formulated with a DCtargeted liposome including LPS as adjuvant (pCIgD-Man-L). It is interesting to note that although no new inoculation was made after $40 \mathrm{dpv}$ in mice, there were high levels of antibody even at $120 \mathrm{dpv}$ with pCIgD-Man-L, but this phenomenon was not observed with pCIgD alone. This can be attributed to the local depot effect, and the selective delivery and activation capacities of the liposomes combined with LPS from B. ovis (Fredriksen and Grip, 2012) thus enhancing and prolonging the exposure of the immune cells to the antigen which results in a specific immune response and long lasting immunity. When isotype antibodies were analyzed, the specific effect of the liposomal vaccine resulted in the increase of the IgG1 levels and the profile of IgG2a, IgG2b when compared with the pCIgD group, where the levels of antibodies were significantly lower. Notably IgG2a is an isotype that is crucial for the defense against viruses. Previous works have shown that IgG2a antibodies are produced in mice upon viral infections (Coutelier et al., 1991) and there are also triggered when DNA vaccines are administered for immunizations (Raz et al., 1996) as a result of T helper 1 cell involvement. This isotype is efficient for complement fixation and acts as an opsonizing agent. It is reported that IFN $\gamma$ can stimulate the IgG2a production both by in vitro and in vivo by activating the B lymphocytes. The increase of IgG2a in the animals treated with our free DNA plasmid suggests that the gD plasmid could be internalized by DCs and that the interaction between DCs-B cells may take place according to CD40:CD40L dependent manner (Bao et al., 2014). The B cell switching was confirmed by the presence of the IgG2a isotype in sera of mice and by the polarization of the T CD4+ cells response to a Th1/Th2 profile.

The immune system fast response to a viral infection confirms the cellular memory to the viral antigens. This could prove that the viral antigens were previously processed by the host and CD4+/CD8+ memory lymphocytes were generated. In this work, we observed that splenocytes in culture from mice immunized with gD plasmids were able to lymphoproliferate against inactivated BoHV-1. Moreover, it is important to highlight that these animals got their last immunization 100 days before their splenocytes were exposed to inactivated BoHV-1. This ability to 
lymphoproliferate in the pCIgD-Man-L group was certainly provided by the adjuvant effect of the targeted liposome because this was not observed with the DNA plasmid administered alone.

We then tested the novel liposome based nanovaccine in a field trial in cattle. When we assessed BoHV-1 specific antibodies in blood the difference was significant between the pCIgD-Man-L immunized group compared to the control group. This increase was also observed in the isotype antibody titers. While in the murine model, the transcription factors in T helper lymphocytes such as GATA-3 associated with enhanced type 2 cytokine gene transcription (IL-4) or T-bet associated to type 1 cytokine (IFN $\gamma$ ) have been widely shown, these factors are not evident in bovines (Estes and Brown, 2002). Bovine IL-4 could induce IgG1 while IFN $\gamma$ may enhance IgG2 over IgG1 (Estes et al., 1995). The results obtained in our study for bovine isotypes elicited by the vaccines, showed increasing levels of IgG1-IgG2 for both vaccines when compared to the control, as it was observed in previous tests with other DNA vaccines or adjuvants (Arulkanthan et al., 1999; Brown et al., 1999; Quattrocchi et al., 2017). However, a significant IgG1:IgG2 ratio increase was observed only for pCIgD-Man-L whereas a significant IgG1 increase solely occurred in the group vaccinated with $\mathrm{pCIgD}$ when compared to the control.

$\operatorname{IgA}$ was assessed from nasal swabs and evaluated 6 days post challenge resulting in higher titer in the pCIgD-Man-L treated group with respect to the control $\mathrm{pCIgD}$ treated group. Reasonably, the persistence and enhanced exposure to glycoprotein D post challenge can be responsible for the expansion of DC-primed cell and IgGs and IgA secreting B cell populations as hypothized by Manoj (Manoj et al., 2004).

CD40 is a co-stimulatory cytokine for various types of cells including B cells, macrophages, non-hematopoietic cells and DCs, for which it has an crucial role in immunity development and in activation of the latter cell types (Elgueta et al., 2009). DCs are the major antigen presenting cells (APC) and they are crucial mediators between immune innate and adaptive responses (Vázquez et al., 2012). When DCs are inactivated, CD40 is expressed constitutively at relatively low levels on their surface (Banchereau et al., 1994). Upon DC cells encounter pathogens, antigens (Kawai and Akira, 2007) or apoptotic cells (Ip and Lau, 2004), CD40 expression is up-regulated on their surface and is activated. In this work, ALDCs were stimulated and activated after incubation with free DNA plasmid and DNA loaded targeted liposomes embedding LPS from Brucella ovis. It is well known that DNA possess CpG domain which can be recognized by APCs and trigger their response (Kuwajima et al., 2006). Here, we observed that ALDCs incubated with free DNA plasmid showed positive up regulation of CD40 molecule. Also, targeted liposomes alone or associated with DNA and functionalized with LPS from Brucella ovis are capable to enhance the expression of CD40 on the surface of DCs. This result proves that the nanovaccines represent a promising platform that comply the idea that an efficient vaccine must target the APCs. Notably, the liposomes formulated without the LPS were not able to activate DCs. In this way, the MANa1-2MAN targeting provides a crucial function to selectively target the dendritic cells while the LPS address its function as an adjuvant of the cell response.

Since it is well known that cell-mediated immunity is very important for virus clearance upon BoHV-1 and other viruses infection, a better protection induced after challenge in animals immunized with targeted DNA loaded liposomes was observed. Animals from pCIgD-Man-L treated group had less viral excretion with significant decrease of viral titer at $5 \mathrm{dpc}$ respect to control group. Furthermore, induction of cellular immune response was observed at 12 $\mathrm{dpc}$ in $\mathrm{pCIgD-Man-L}$ treated group with positive response to viral-specific PBMCs proliferation. The activation of cellular immune response could be correlated with the enhancement of IgG1 isotype (Sin et al., 1999). The increase of 
the lymphoproliferative effect in pCIgD-Man-L treated group shows a better immune response, in which memory cells became established and were able to recognize the viral antigen more quickly than in pCIgD treated and control groups.

Although, the clinical score was similar for the animals vaccinated with free DNA plasmid and those immunized with the DNA loaded targeted liposomes, these animals presented less clinical symptoms than the control animals during viremia.

The development of efficient vaccines for BoHV-1 based on selective DNA delivery to DCs using new carriers including liposomes may allow better control of virus circulation in livestock. Although this trial as a proof of concept demonstrates that animals vaccinated with the engineered liposomal formulation improved humoral and cellular response levels, the impact of formulation parameters including vaccine dose and booster/lipid ratio still need to be systematically elucidated. Nevertheless, this study opens up perspectives for the generation of a new generation vaccine for robust protection of the animals against viral exposure.

To our knowledge, it is the first time that liposomes decorated with MAN 1 1-2MAN-PEG-DOPE as immune targeting agent were used in a DNA based vaccine for BoHV-1 immunization and tested in cattle.

\section{Acknowledgments}

The authors wish to thank Mr. Osvaldo Zabal and Ms. Marta D'Angelo for technical assistance in tissue culture, Ms. Pamela Angeletti for laboratory technical assistance, Dr. Gabriel Pinto, Ms. Fátima Torales and Ms. Sandra Rivarola for technical assistance in biotherium, Mgter. Carlos Robles for the LPS of B. ovis, INTA Balcarce for kindly supplying the animals, Mr. Martín Mayoral and Mr. Walter Bagazette for the care of the bovines.

\section{Funding Satetment}

This work was supported by INTA project PNBIO 1131032 and by PICT2014-0891 grant from Agencia Nacional de Promoción Científica y Tecnológica, Ministry of Science and Technology, Argentina.

\section{Ethics Statement}

The authors confirm that the ethical policies of the journal, as noted on the journal's author guidelines page, have been adhered to and the appropriate ethical review committee approval has been received.

The Institutional Committee for the Care and Use of Experimental Animals, CICUAE-INTA Castelar, and CICUAE INTA-CeRBAS, Balcarce, Argentina, has given its approval for work with mice (44/2018) and cattle (123/2017) respectively.

\section{Conflict of Interest Statement}

The authors declare no conflict of interest.

\section{Data Availability Statement}


The data supporting the findings of this study are available on request from the corresponding author.

\section{References}

Arulkanthan, A., W.C. Brown, T.C. McGuire, and D.P. Knowles, 1999: Biased immunoglobulin G1 isotype responses induced in cattle with DNA expressing mspla of Anaplasma marginale. Infect. Immun. 67, 3481-7.

Babiuk, L.A., J. L'Italien, S. van Drunen Littel-van den Hurk, T. Zamb, M.J.P. Lawman, G. Hughes, and G.A. Gifford, 1987: Protection of cattle from bovine herpesvirus type I (BHV-1) infection by immunization with individual viral glycoproteins. Virology 159, 57-66, DOI: 10.1016/0042-6822(87)90347-3.

Banchereau, J., F. Bazan, D. Blanchard, F. Briè, J.P. Galizzi, C. van Kooten, Y.J. Liu, F. Rousset, and S. Saeland, 1994: The CD40 Antigen and its Ligand Vol. 12Annu. Rev. Immunol.

Bao, Y., X. Liu, C. Han, S. Xu, B. Xie, Q. Zhang, Y. Gu, J. Hou, L. Qian, C. Qian, H. Han, and X. Cao, 2014: Identification of IFN- $\gamma$-producing innate B cells. Cell Res. 24, 161-76, DOI: 10.1038/cr.2013.155.

Bitsch, V., 1973: Infectious bovine rhinotracheitis virus infection in bulls, with special reference to preputial infection. Appl. Microbiol. 26, 337-43.

Blickenstorfer, S., M. Engels, C. Guerdat, C. Saucy, M. Reist, H. Schwermer, and L. Perler, 2010: [Infectious bovine rhinotracheitis (IBR) in the canton of Jura: an epidemiological outbreak investigation]. Schweiz. Arch. Tierheilkd. 152, 555-60, DOI: 10.1024/0036-7281/a000124.

Blome, S., C. Gabriel, and M. Beer, 2013: [Possibilities and limitations in veterinary vaccine development using the example of classical swine fever]. Berl. Munch. Tierarztl. Wochenschr. 126, 481-490, DOI: 10.2376/00059366-126-41.

Brown, W.C., C.E. Suarez, L.K.M. Shoda, and D.. Estes, 1999: Modulation of host immune responses by protozoal DNA. Vet. Immunol. Immunopathol. 72, 87-94, DOI: 10.1016/S0165-2427(99)00120-8.

Coutelier, J.P., J.T. van der Logt, and F.W. Heessen, 1991: IgG subclass distribution of primary and secondary immune responses concomitant with viral infection. J. Immunol. 147, 1383-6.

Deshpande, M.S., T.C. Ambagala, N.R. Hegde, M.J. Hariharan, M. Navaratnam, and S. Srikumaran, 2002: Induction of cytotoxic T-lymphocytes specific for bovine herpesvirus-1 by DNA immunization. Vaccine 20, 3744-51, DOI: 10.1016/s0264-410x(02)00375-4.

Di Giacomo, S., V. Quattrocchi, and P. Zamorano, 2015: Use of Adjuvants to Enhance the Immune Response Induced by a DNA Vaccine Against Bovine Herpesvirus-1. Viral Immunol. 28, 343-346, DOI: 10.1089/vim.2014.0113.

Elgueta, R., M.J. Benson, V.C. De Vries, A. Wasiuk, Y. Guo, and R.J. Noelle, 2009 ( May): Molecular mechanism and function of CD40/CD40L engagement in the immune system. Immunol. Rev. 229, 152-172, DOI: 10.1111/j.1600-065X.2009.00782.x. NIH Public Access.

Estes, D.M., and W.C. Brown, 2002 ( November): Type 1 and type 2 responses in regulation of Ig isotype expression in cattle. Vet. Immunol. Immunopathol. 90, 1-10, DOI: 10.1016/S0165-2427(02)00201-5.

Estes, D.M., A. Hirano, V.T. Heussler, D.A. Dobbelaere, and W.C. Brown, 1995: Expression and biological activities of bovine interleukin 4: effects of recombinant bovine interleukin 4 on $\mathrm{T}$ cell proliferation and B cell differentiation and proliferation in vitro. Cell. Immunol. 163, 268-79, DOI: 10.1006/cimm.1995.1126.

Feinberg, H., R. Castelli, K. Drickamer, P.H. Seeberger, and W.I. Weis, 2007: Multiple modes of binding enhance the 
affinity of DC-SIGN for high mannose N-linked glycans found on viral glycoproteins. J. Biol. Chem. 282, 4202-4209, DOI: 10.1074/jbc.M609689200.

Fredriksen, B.N., and J. Grip, 2012: PLGA/PLA micro- and nanoparticle formulations serve as antigen depots and induce elevated humoral responses after immunization of Atlantic salmon (Salmo salar L.). Vaccine 30, 656-67, DOI: $10.1016 /$ j.vaccine.2011.10.105.

Fuller, A.O., and W.C. Lee, 1992: Herpes simplex virus type 1 entry through a cascade of virus-cell interactions requires different roles of $\mathrm{gD}$ and $\mathrm{gH}$ in penetration. J. Virol. 66, 5002-5012, DOI: 10.1128/jvi.66.8.50025012.1992.

Gregoriadis, G., A. Bacon, W. Caparros-Wanderley, and B. McCormack, 2002 (20. December): A role for liposomes in genetic vaccination. Vaccine 20, B1-B9, DOI: 10.1016/S0264-410X(02)00514-5. Elsevier BV.

Hope, J.C., C.J. Howard, H. Prentice, and B. Charleston, 2006: Isolation and purification of afferent lymph dendritic cells that drain the skin of cattle. Nat. Protoc. 1, 982-7, DOI: 10.1038/nprot.2006.125.

Huang, Y., L.A. Babiuk, and S. van Drunen Littel-van den Hurk, 2005: Immunization with a bovine herpesvirus 1 glycoprotein B DNA vaccine induces cytotoxic T-lymphocyte responses in mice and cattle. J. Gen. Virol. 86, 887-898, DOI: 10.1099/vir.0.80533-0.

Ip, W.-K., and Y.-L. Lau, 2004: Distinct Maturation of, but Not Migration between, Human Monocyte-Derived Dendritic Cells upon Ingestion of Apoptotic Cells of Early or Late Phases. J. Immunol. 173, 189-196, DOI: 10.4049/jimmunol.173.1.189.

Jones, C., 2003 ( January): Herpes simplex virus type 1 and bovine herpesvirus 1 latency. Clin. Microbiol. Rev. 16, 79-95, DOI: 10.1128/CMR.16.1.79-95.2003. Clin Microbiol Rev.

Kanthesh, M., N. Loide, N. Raghu, T.S. Gopenath, G.K. Chandrashekrappa, K. Murugesan, G. Ashok, and K. Gobianand, 2018: DNA Vaccines. Medwin Publ. 3.

Kawai, T., and S. Akira, 2007 (1. November): Signaling to NF-кB by Toll-like receptors. Trends Mol. Med. 13, 460469, DOI: 10.1016/j.molmed.2007.09.002. Elsevier.

Krieg, A.M., A.K. Yi, S. Matson, T.J. Waldschmidt, G.A. Bishop, R. Teasdale, G.A. Koretzky, and D.M. Klinman, 1995: CpG motifs in bacterial DNA trigger direct B-cell activation. Nature 374, 546-9, DOI: 10.1038/374546a0.

Kuwajima, S., T. Sato, K. Ishida, H. Tada, H. Tezuka, and T. Ohteki, 2006: Interleukin 15-dependent crosstalk between conventional and plasmacytoid dendritic cells is essential for CpG-induced immune activation. Nat. Immunol. 7, 740-6, DOI: 10.1038/ni1348.

Langellotti, C.A., J.S. Pappalardo, V. Quattrocchi, C. Mongini, and P. Zamorano, 2011: Induction of specific cytotoxic activity for bovine herpesvirus-1 by DNA immunization with different adjuvants. Antiviral Res. 90, 134-42, DOI: 10.1016/j.antiviral.2011.03.185.

Lee, S.H., S.N. Danishmalik, and J.I. Sin, 2015: DNA vaccines, electroporation and their applications in cancer treatment. Hum. Vaccines Immunother. 11, 1889-1900, DOI: 10.1080/21645515.2015.1035502.

Lewis, P.J., van Drunen Littel-van den Hurk, and L.A. Babiuk, 1999: Altering the cellular location of an antigen expressed by a DNA-based vaccine modulates the immune response. J. Virol. 73, 10214-23.

Ludewig, B., F. Barchiesi, M. Pericin, R.M. Zinkernagel, H. Hengartner, and R.A. Schwendener, 2000: In vivo 
antigen loading and activation of dendritic cells via a liposomal peptide vaccine mediates protective antiviral and anti-tumour immunity. Vaccine 19, 23-32, DOI: 10.1016/S0264-410X(00)00163-8.

Manoj, S., P.J. Griebel, L.A. Babiuk, and S. van Drunen Littel-van den Hurk, 2004: Modulation of immune responses to bovine herpesvirus-1 in cattle by immunization with a DNA vaccine encoding glycoprotein D as a fusion protein with bovine CD154. Immunology 112, 328-38, DOI: 10.1111/j.1365-2567.2004.01877.x.

Mars, M.H., M.C.M. De Jong, P. Franken, and J.T. Van Oirschot, 2001: Efficacy of a live glycoprotein E-negative bovine herpesvirus 1 vaccine in cattle in the field. Vaccine 19, 1924-1930, DOI: 10.1016/S0264$410 \mathrm{X}(00) 00435-7$.

Miethke, A., G.M. Keil, F. Weiland, and T.C. Mettenleiter, 1995: Unidirectional complementation between glycoprotein B homologues of pseudorabies virus and bovine herpesvirus 1 is determined by the carboxyterminal part of the molecule. J. Gen. Virol. 76 ( Pt 7), 1623-35, DOI: 10.1099/0022-1317-76-7-1623.

Pappalardo, J.S., M.M. Chodilef, A.C. Mignaqui, R. Marcellino, A.L. Zamit, S. Salmaso, T.S. Levchenko, V.P. Torchilin, and C.A. Robles, 2016: Novel mannose- $\alpha(1 \rightarrow 2)$-mannose decorated liposomes for dendritic cell targeted nanovaccines: strong humoral and cell immune response against Brucella ovis. p. 33. In: Int. Congr. Immunol. Vol. 46, Suppl. 1 Aust. Melbourne: European Journal of Immunology.

Pappalardo, J.S., M. Toniutti, S. Salmaso, T.S. Levchenko, and V.P. Torchilin, 2013: Compounds and methods for targeted immune system delivery (Granted: 2018.05.08) United States Patent and Trademark Office (USPTO), ed. World Intellectual Property Organization (WIPO). United States of America.

Quattrocchi, V., I. Soria, C.A. Langellotti, V. Gnazzo, M. Gammella, D.P. Moore, and P.I. Zamorano, 2017: A DNA vaccine formulated with chemical adjuvant provides partial protection against bovine herpes virus infection in cattle. Front. Immunol. 8, DOI: 10.3389/fimmu.2017.00037.

Rajčáni, J., T. Moško, and I. Režuchová, 2005: Current developments in viral DNA vaccines: shall they solve the unsolved? Rev. Med. Virol. 15, 303-325, DOI: 10.1002/rmv.467.

Raz, E., H. Tighe, Y. Sato, M. Corr, J.A. Dudler, M. Roman, S.L. Swain, H.L. Spiegelberg, and D.A. Carson, 1996: Preferential induction of a Th1 immune response and inhibition of specific IgE antibody formation by plasmid DNA immunization. Proc. Natl. Acad. Sci. U. S. A. 93, 5141-5145, DOI: 10.1073/pnas.93.10.5141.

Reed, L.J., and H. Muench, 1938: A simple method of estimating fifthy percent endpoints. Am. J. Epidemiol. 27, 493497, DOI: 10.1093/oxfordjournals.aje.a118408.

Robles, C., 2009: Protocolo para la obtención del antígeno HS (Hot Saline Extract) de Brucella Ovis Cepa Reo 198 para el diagnóstico serológico de la brucelosis ovina. Comunicación Técnica EEA Bariloche Nro. 557. 2009., Bariloche.

Romera, S.A., L.A. Hilgers, M. Puntel, P.I. Zamorano, V.L. Alcon, M.J. Dus Santos, J. Blanco Viera, M. V Borca, and A.M. Sadir, 2000: Adjuvant effects of sulfolipo-cyclodextrin in a squalane-in-water and water-in-mineral oil emulsions for BHV-1 vaccines in cattle. Vaccine 19, 132-41, DOI: 10.1016/s0264-410x(00)00104-3.

Romera, S.A., M. Puntel, V. Quattrocchi, P.D.M. Zajac, P. Zamorano, J. Blanco Viera, C. Carrillo, S. Chowdhury, M. V. Borca, and A.M. Sadir, 2014: Protection induced by a glycoprotein E-deleted bovine herpesvirus type 1 marker strain used either as an inactivated or live attenuated vaccine in cattle. BMC Vet. Res. 10, 8, DOI: $10.1186 / 1746-6148-10-8$. 
Sin, J.I., J.J. Kim, R.L. Arnold, K.E. Shroff, D. McCallus, C. Pachuk, S.P. McElhiney, M.W. Wolf, S.J. Pompa-de Bruin, T.J. Higgins, R.B. Ciccarelli, and D.B. Weiner, 1999: IL-12 gene as a DNA vaccine adjuvant in a herpes mouse model: IL-12 enhances Th1-type CD4+ T cell-mediated protective immunity against herpes simplex virus-2 challenge. J. Immunol. 162, 2912-21.

Suschak, J.J., J.A. Williams, and C.S. Schmaljohn, 2017 (2. December): Advancements in DNA vaccine vectors, nonmechanical delivery methods, and molecular adjuvants to increase immunogenicity. Hum. Vaccines Immunother. 13, 2837-2848, DOI: 10.1080/21645515.2017.1330236. Taylor and Francis Inc.

Tikoo, S.K., M. Campos, and L.A. Babiuk, 1995: Bovine herpesvirus 1 (Bhv-1): Biology, Pathogenesis, and Control. Adv. Virus Res. 45, 191-223, DOI: 10.1016/S0065-3527(08)60061-5.

Van Drunen Littel-van den Hurk, R.P. Braun, P.J. Lewis, B.C. Karvonen, M.E. Baca-Estrada, M. Snider, D. McCartney, T. Watts, and L.A. Babiuk, 1998: Intradermal immunization with a bovine herpesvirus-1 DNA vaccine induces protective immunity in cattle. J. Gen. Virol. 79 ( Pt 4), 831-9, DOI: 10.1099/0022-1317-79-4831.

van Drunen Littel-van den Hurk, S., 2006: Rationale and perspectives on the success of vaccination against bovine herpesvirus-1. Vet. Microbiol. 113, 275-82, DOI: 10.1016/j.vetmic.2005.11.002.

Vázquez, M.B., M. Sureda, and J. Rebollo, 2012 (1. January): Dendritic cells I: Basic biology and functions. Inmunologia 31, 21-30, DOI: 10.1016/j.inmuno.2011.10.001. Elsevier Doyma.

Yamakawa, Y., C. Pennelegion, S. Willcocks, A. Stalker, N. MacHugh, D. Burt, T.J. Coffey, and D. Werling, 2008: Identification and functional characterization of a bovine orthologue to DC-SIGN. J. Leukoc. Biol. 83, 13961403, DOI: $10.1189 /$ jlb.0807523.

Yamamoto, S., T. Yamamoto, T. Kataoka, E. Kuramoto, O. Yano, and T. Tokunaga, 1992: Unique palindromic sequences in synthetic oligonucleotides are required to induce IFN [correction of INF] and augment IFNmediated [correction of INF] natural killer activity. J. Immunol. 148, 4072-6.

Zaman, M., M.F. Good, and I. Toth, 2013: Nanovaccines and their mode of action. Methods 60, 226-31, DOI: 10.1016/j.ymeth.2013.04.014.

Zamorano, P., O. Taboga, M. Domínguez, S.A. Romera, M. Puntel, C. Tami, C. Mongini, C. Waldner, E. Palma, and A. Sadir, 2002: BHV-1 DNA vaccination: Effect of the adjuvant RN-205 on the modulation of the immune response in mice. Vaccine 20, 2656-2664, DOI: 10.1016/S0264-410X(02)00211-6.

Zonouzi, A.A., A.P. Zonouzi, and M. Abkar, 2016: Recent Applications of DNA Vaccines in Cancer Therapy Vol. 2016Mol. Med. J. Kashan, Iran.

This article is protected by copyright. All rights reserved 

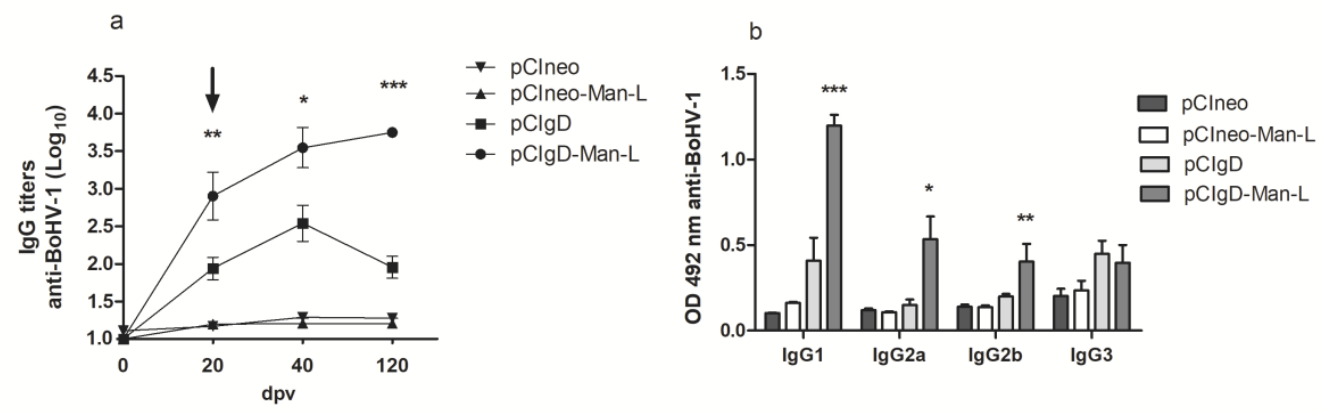

tbed_13718_f1.tif 


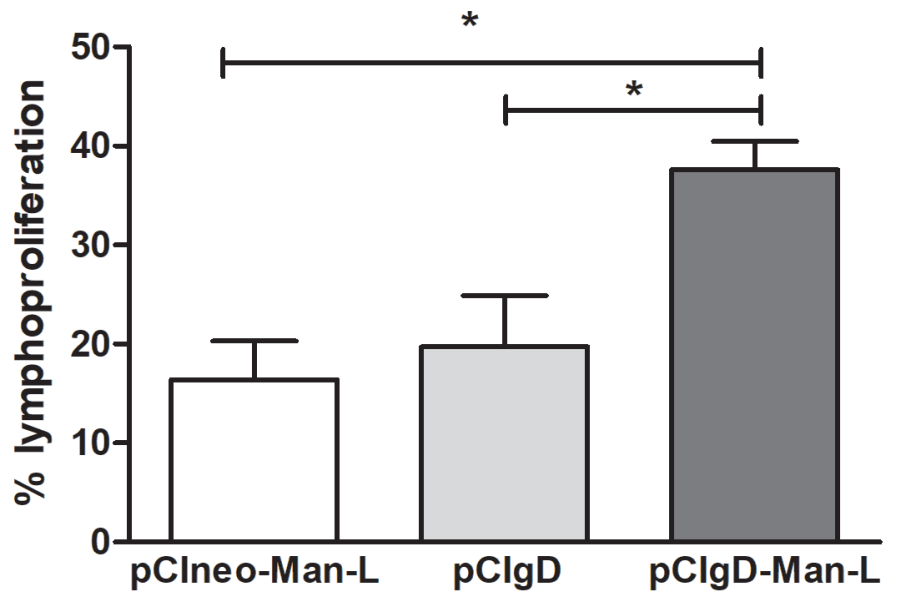

tbed_13718_f2.tif 

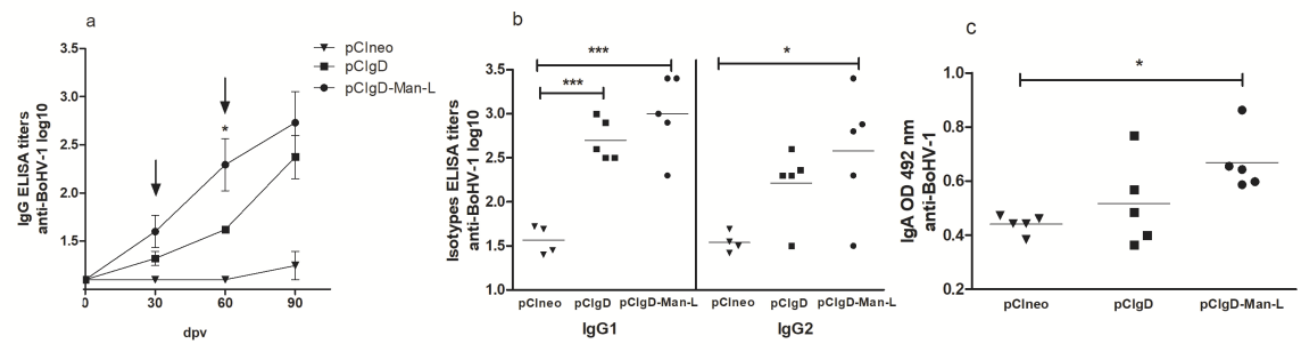

tbed_13718_f3.tif 


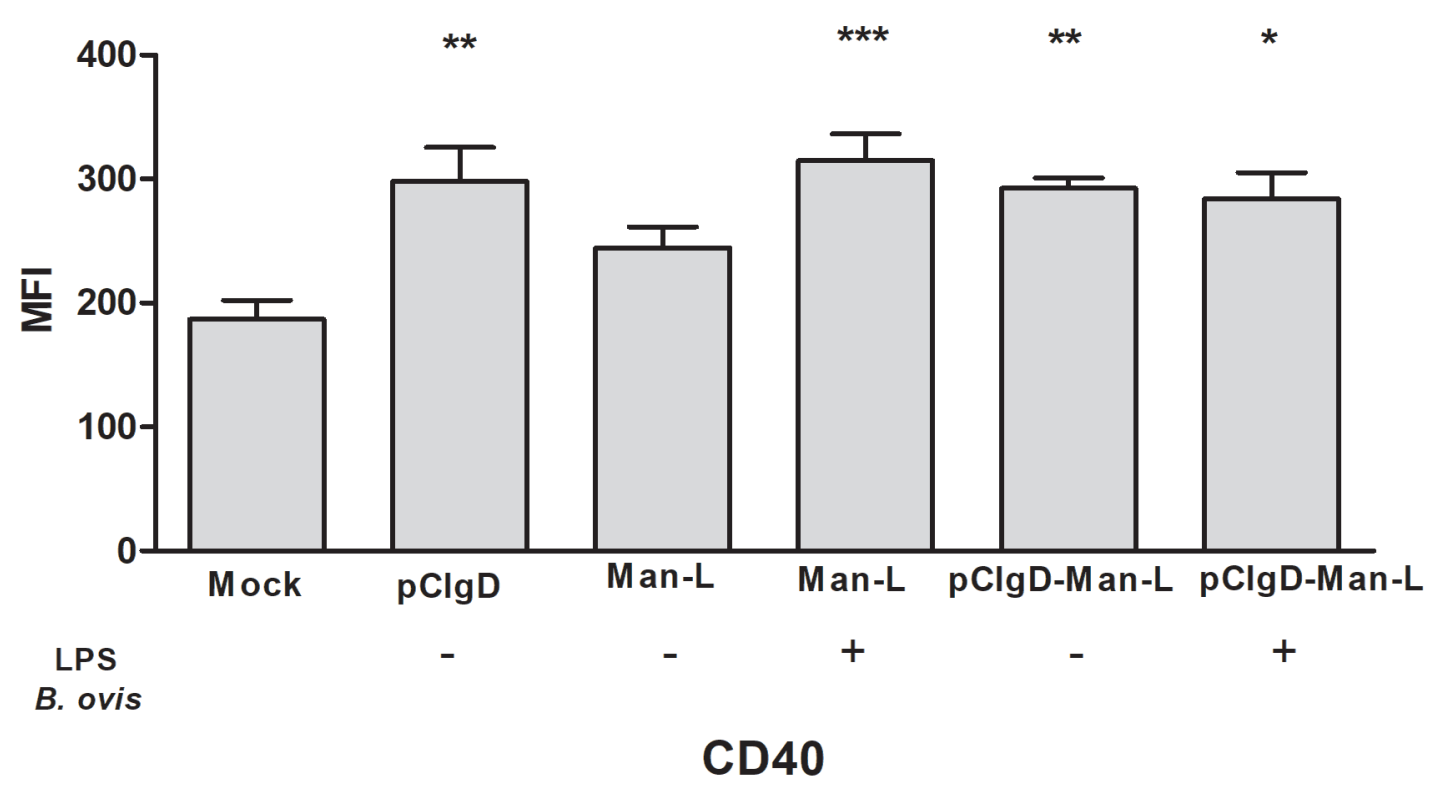

tbed_13718_f4.tif 


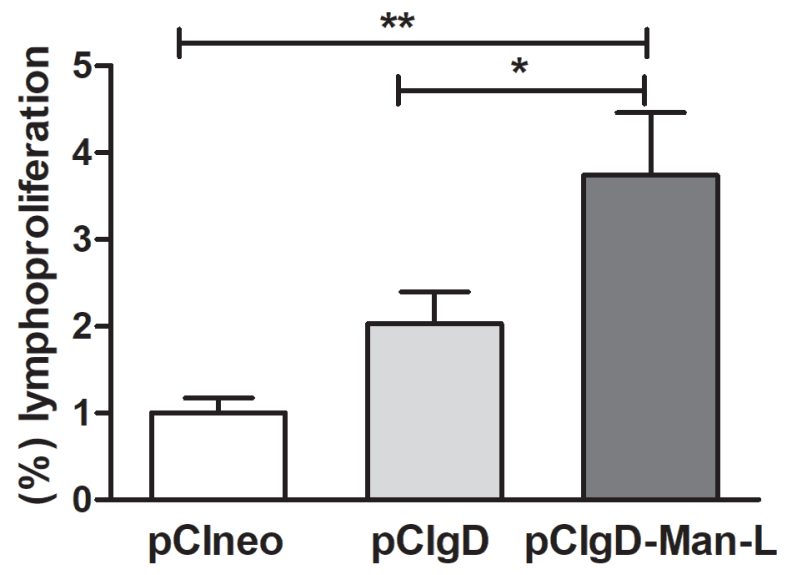

tbed_13718_f5.tif 


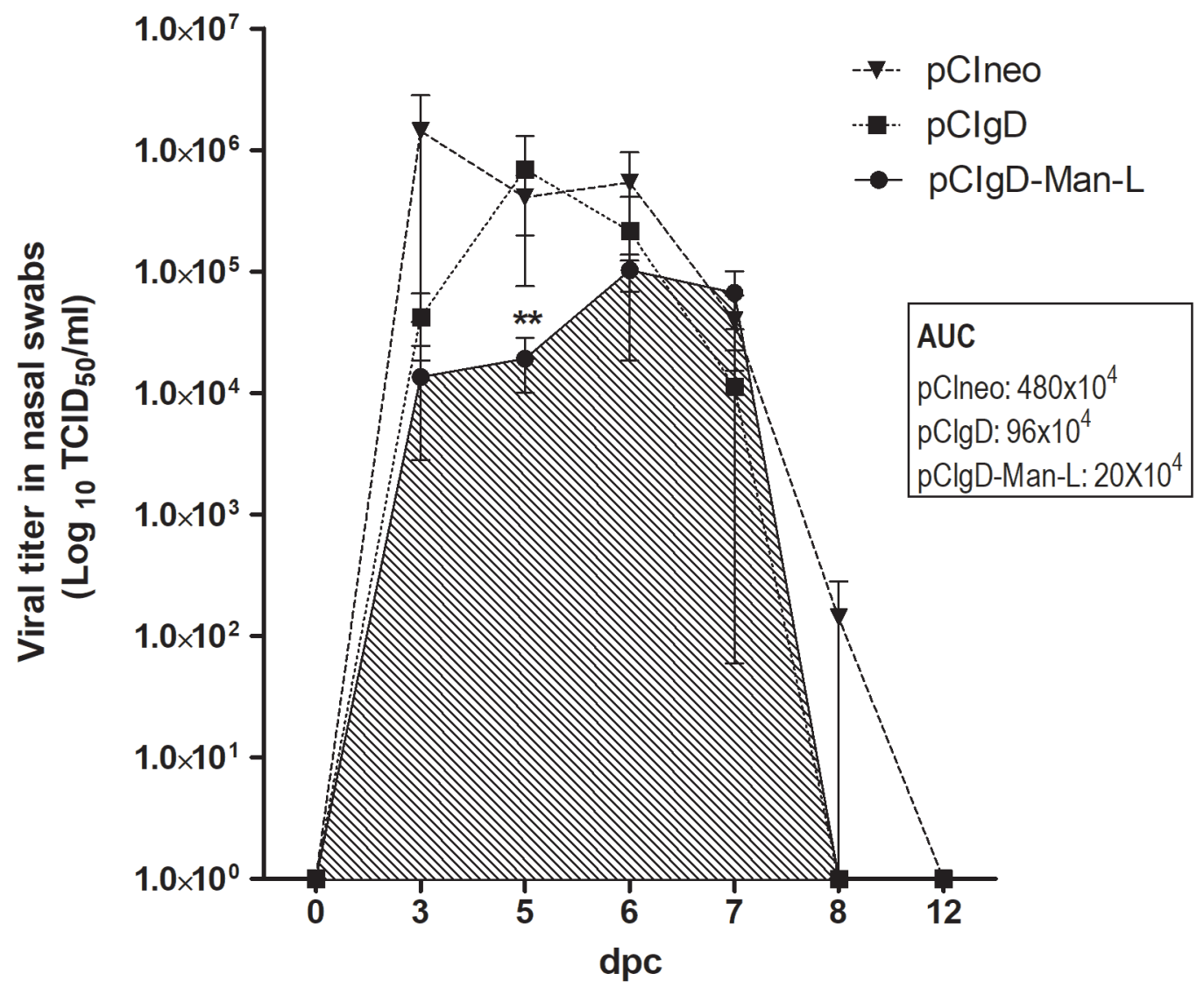

tbed_13718_f6.tif 


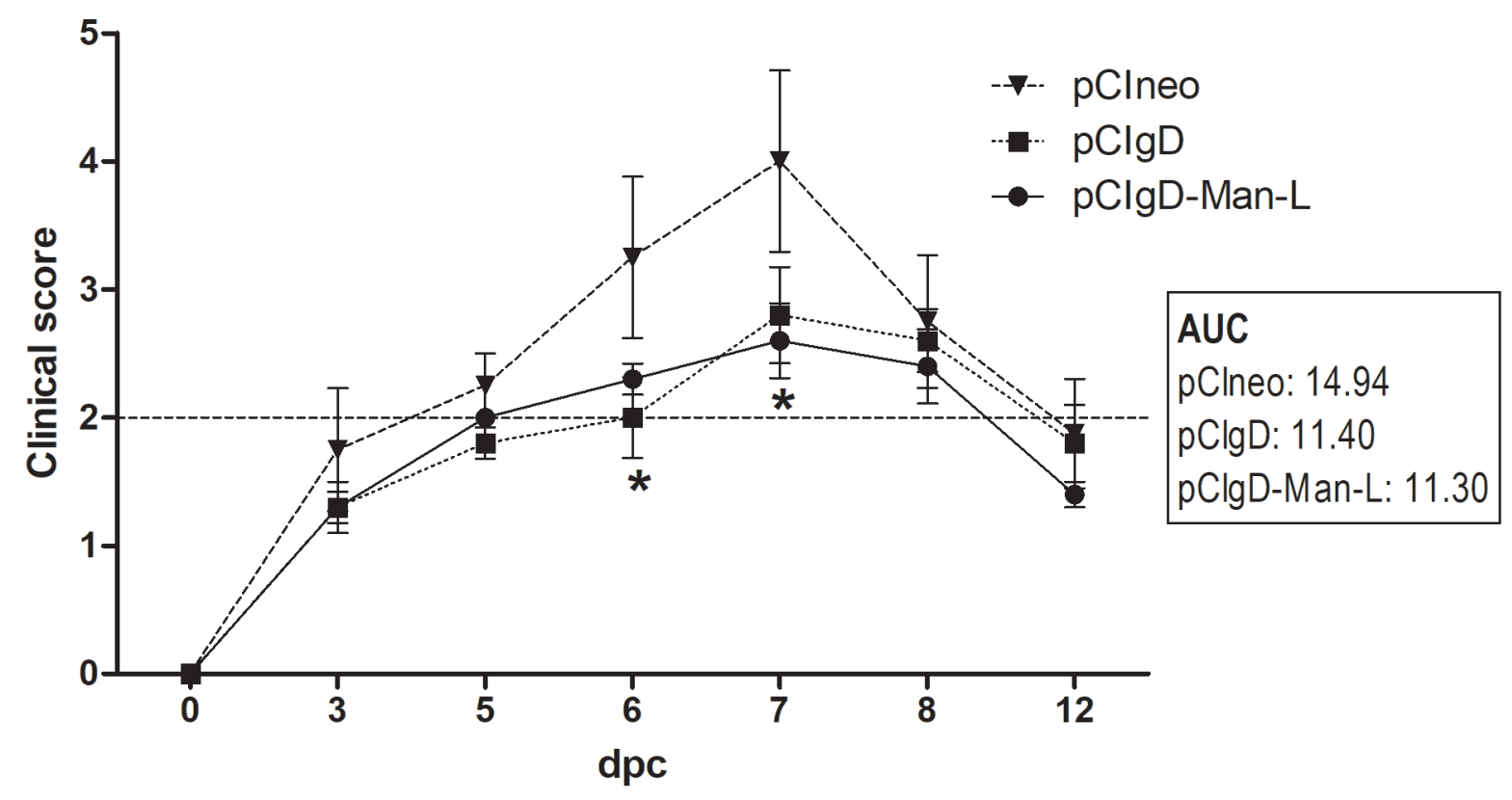

tbed_13718_f7.tif

This article is protected by copyright. All rights reserved 